\title{
INFECTIONS DURING FIRST YEAR OF RENAL TRANSPLANTATION: ETIOLOGY, RISK FACTORS AND OUTCOIME
}

KEY WORDS: post renal transplant infection; Graft dysfunction; etiology

\section{Dr. Amit Katyal*}

\section{Dr. M.M.} Bahadur
Assistant Professor, Department of Medicine, Army Hospital (R\&R), Delhi*Corresponding Author

\section{Director of Nephrology, Department of Nephrology,Jaslok Hospital, Mumbai}

Background: Renal transplantation is the best treatment in managing end stage renal disease patients.However infections in these patients remain the leading cause of morbidity and mortality[1].Various factors like age, co-morbid infections like Hepatitis $\mathbf{C}$ infection and presence of Diabetes Mellitus, play a role in development of these infections. In developing country like ours, the spectrum of infection is likely to be different from the western world[2]. There is paucity of data on this aspect. There exists a conflict in literature regarding the predisposition to these infections and their impact on graft outcome.

Aims \& Objectives : This study proposes to analyse the predisposing factors, spectrum of infections in renal transplant recipients and their impact on graft function.

Materials \& Methods : Hundred renal transplant patients who received transplant between 01 Jan 2015 to Dec 2015 were prospectively followed for a period of one year for development of a major infection. All patients underwent thorough evaluation with complete blood count, urine and blood cultures, Radiological investigations and invasive investigations were done on case to case basis to achieve an etiological diagnosis. Special investigations were done when clinically indicated and infections were diagnosed based on established criteria. Those patients who had evidence of graft dysfunction were subjected to kidney biopsy. Descriptive analysis was done for all variables statistical analysis was done using paired/unpaired T test. A p value of < 0.05 was considered significant.

Results: 68 patients (68\%) had 138 episodes of infection (i.e. $2.02 /$ patient)[3]. There were $42 \%$ episodes of bacterial infections, $29 \%$ of viral infections, $8.7 \%$ of fungal, $7.1 \%$ tubercular and $14.4 \%$ had miscellaneous infection. There was no significant correlation between development of infection and variables like Diabetes Mellitus, age and HCV infection. There was significant increase in creatinine value at the end of one year, in the patients of infection(p value0.003), which on comparison with the non infected group was not significant $(\mathrm{p}>0.05)$.

Conclusion: Nearly $68 \%$ of transplant recipients had an episode of major infection in the first year of transplantation.The majority of infection were bacterial(42\%); and the dominant amongst them, was UTI. Graft survival was not inferior in these patients, at the end of one year.

\section{Introduction:}

Renal transplant is the cheapest and best chance of rehabilitating individuals with end stage renal disease. Advances in surgical techniques, immunobiology and immunosuppression has resulted in significant improvement in patient and graft outcome.Today most centres[4] have short term patient and graft survival of over $95 \%$. The short term survival, i.e. 1 year after the renal transplantation, has considerably improved than in the past and mainly due to decline in mortality associated with infectious complications. Although the infection-related mortality rates have decreased from $50 \%$ to $5 \%$ over last 20 years, this remains a serious complication after transplantation, particularly since post-transplant infections have changed in epidemiology. Immunosuppression plays a major role in the causation of infections and the incidence of infectious complications after kidney transplantation has increased in last decade due to availability of potent immunosuppressive agents. Most infections occur early in the post-transplantation course, with about two third of renal transplant recipients (RTR), experiencing an infection related complication, in the first year after transplantation[5]. In developing countries, infections claim more post-transplantation lives than graft failure from rejection or cardiovascular disease[6]. The risk for death in transplant population doubles over a 10-year period if there is a major infection[7].

Material and Methods: This study entails the etiology and risk factors of major infections in hospitalised renal transplant recipients during the first year of transplantation and its impact on graft outcome. Hundred renal transplant patients who received transplant from Jan. 2015 to Dec. 2015 in this tertiary care transplant centre in Western India, were prospectively followed up for a period of one year, for development of major infection. In all patients presenting with fever detailed history was taken and examination was done. Major infection was defined as: where patient requires hospitalisation for extensive evaluation/treatment, which is not possible on outpatient basis. Informed consent of all the participants was taken. The infectious events were identified through the observation of clinical signs and symptoms; and also through complementary laboratory assessment. Urinary tract infection (UTI) was defined as: the presence of dysuria and/or pyuria, accompanied by fever, with positive or negative urine culture. Cytomegalovirus infection (CMV) was defined as: the presence of fever, abdominal pain, diarrhoea, and myalgia, accompanied or not by anemia, leukopenia; confirmed by positive antigenemia in circulating blood (detected by PCR); or the histopathologic evidence of the characteristic cytomegalic inclusions in patients undergoing biopsy of the gastrointestinal tract mucosa or of pulmonary tissue. Herpes zoster infection was defined as: the presence of vesicular, vesico-pustular or vesico-ulcerated lesions, with hyperemic ulcer base, in body regions delineated by specific dermatomes. Pulmonary infections were defined as: the presence of respiratory insufficiency of any severity, accompanied by fever and compatible physical examination and radiologic study; with compatible findings on sputum microscopy, with/without positive culture of bronchial aspirate. Bloodstream infection was defined as: the positive culture of peripheral blood (obtained on multiple sampling) with/without fever, with/without central line tip culture, with/without other clinical manifestations like hypotension, tachycardia etc.Allograft dysfunction was defined as: elevation of serum creatinine value by more than $0.3 \mathrm{mg} / \mathrm{dl}$. All patients received ampicillin + cloxacillin as antibiotic prophylaxis perioperatively and postoperatively. Treatment of the infections was according to clinical judgment of the 
medical team, and culture findings. Variables to be considered included - age,Diabetes Mellitus; and hepatitis C prior to transplantation. Data analysis is done with SPSS Software ver. 15 and Sigmaplot ver.11. Quantitative data is presented with the help of Mean, Standard Deviation, Median and IQR. Comparison between study groups is done with the help of Unpaired T test. Qualitative data is presented with the help of Frequency and Percentage table, association among study group is assessed with the help of Chi-Square test. Pvalue less than 0.05 is considered significant.

\section{Results:}

The baseline characteristics of the study population is given in Table 1. All patients of Hepatitis B/C became seronegative before transplant with antiviral therapy.The majority of the patients received induction therapy with Inj Basiliximab; and maintenance immunosuppression with Tab Tacrolimus, Mycophenolate Mofetil and Prednisolone.

\section{Table 1. Baseline Characteristics}

\begin{tabular}{|c|c|c|c|}
\hline Total Patients & & \multicolumn{2}{|l|}{100} \\
\hline \multirow[t]{4}{*}{ Age (in years) } & Range & \multicolumn{2}{|l|}{09 to 53} \\
\hline & Mean & \multicolumn{2}{|l|}{32} \\
\hline & & \multicolumn{2}{|c|}{ Study Sample } \\
\hline & & in number & in $\%$ \\
\hline \multirow[t]{2}{*}{ Recipient } & Male & 80 & 80 \\
\hline & Female & 20 & 20 \\
\hline \multirow[t]{2}{*}{ Donor } & Male & 30 & 30 \\
\hline & Female & 70 & 70 \\
\hline \multirow{5}{*}{$\begin{array}{c}\text { Native Kidney } \\
\text { disease }\end{array}$} & CGN & 44 & 44 \\
\hline & CIN & 18 & 18 \\
\hline & $\overline{\mathrm{DN}}$ & 30 & 30 \\
\hline & ADPKD & 6 & 6 \\
\hline & $\begin{array}{l}\text { Chronic } \\
\text { rejection }\end{array}$ & 2 & 2 \\
\hline \multirow[t]{2}{*}{ Serology } & $\mathrm{HCV}$ & 8 & 8 \\
\hline & HBsAg & 21 & 2 \\
\hline Diabetes & Yes & 36 & 36 \\
\hline Mellitus/NODAT & No & 64 & 64 \\
\hline
\end{tabular}

Spectrum of infections: 100 patients developed 138 episodes of major infection i.e. a mean of 1.38 episode/patient. Out of the 100 patients, 68 developed single/multiple infections. The mean age of the patient who had infection was 31.24 years (range 16-54 years); and those who did not have was 31.28 years.There were 58 episodes(42.02\%) of bacterial infections, 40 episodes $(28.98 \%)$ of viral infections, eight episodes of tuberculosis( $5.79 \%)$, twelve episodes of fungal infection( $8.69 \%)$ and 20 episodes(14.49\%), due to miscellaneous reasons[Chart 1]. Bacterial infections: 28 patients developed UTI with 42 episodes of infection(30.43\%). 12 patients developed Pneumonitis, constituting $8.69 \%$ of episodes of all infections. Four patients developed bacteremias ( $2.89 \%$ of all episodes of infections), out of which one died. Viral Infection:There were 40 episodes of viral infection; with 12 patients of chicken pox, constituting $8.69 \%$ of episodes of all infections; eight patients developed Herpes zoster and BK virus :5.8\% of all episodes of infections. One patient of Herpes zoster had dissemination of disease and had a successful outcome. Four patients developed CMV infection(2.9\%) of which two died ;eight patients developed TB(7.14\%) with average time from transplant of three months. Fungal infections: Twelve patients $(8.69 \%)$ had evidence of candidiasis demonstrated on Upper GI endoscopy.

Miscellaneous causes: Twenty patients(14.49\%) included 14 of gastroenteritis, four bronchitis; and two malaria. The etiology of bacterial and viral infections is shown in Chart 2 \& 3 , respectively.
Risk Factors: Five variables; Age, Diabetes Mellitus, Hepatitis C, Type of immunosuppression \& ABO compatibility; were correlated with development of infections as in Table 2 below. Chart 1: Spectrum of infections

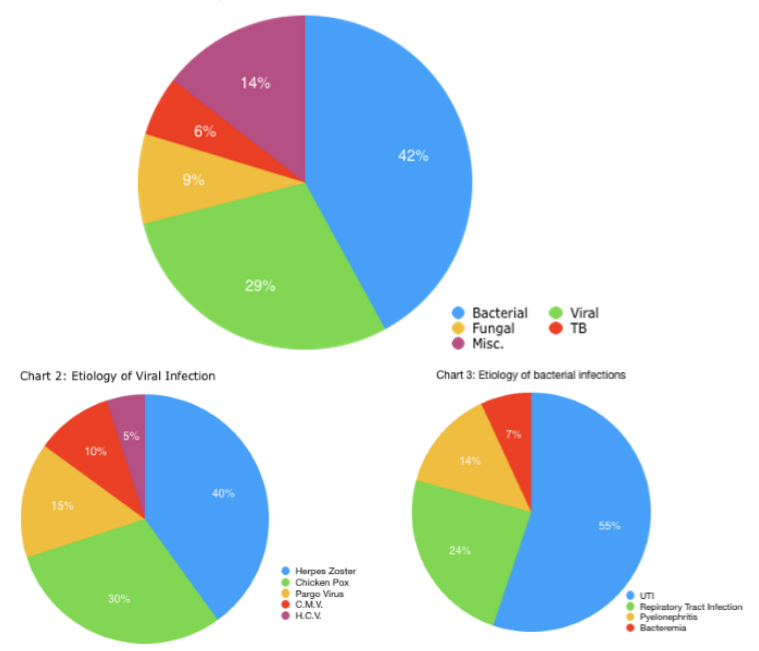

Risk Factors: Five variables; Age, Diabetes Mellitus Hepatitis C, Type of immunosuppression \& ABO compatibility; were correlated with development of infections as in Table 2 below.

\section{Table 2: Risk Factors of Major Infections}

\begin{tabular}{|c|c|c|c|c|c|c|}
\hline \multicolumn{2}{|c|}{} & $\begin{array}{c}\text { Cases } \\
\text { (of 68) }\end{array}$ & $\begin{array}{c}\text { Control } \\
\text { (of 32) }\end{array}$ & $\begin{array}{c}\text { p- } \\
\text { value }\end{array}$ \\
\hline \multirow{2}{*}{ AGE (in years) } & $<=40$ & 45 & & 28 & & 0.29 \\
\cline { 2 - 7 } & $>40$ & 23 & & 4 & & \\
\hline Diabetes Mellitus & Yes & 22 & & 12 & & 0.23 \\
\cline { 2 - 7 } & No & 46 & & 20 & & \\
\hline \multirow{2}{*}{ HCV } & Yes & 8 & & 2 & & 0.69 \\
\cline { 2 - 7 } & No & 60 & & 30 & & \\
\hline \multirow{2}{*}{$\begin{array}{c}\text { Type of } \\
\text { Timmosuppression }\end{array}$} & TMP & 64 & & 30 & 0.76 \\
\hline & CMP & 4 & & 2 & & \\
\hline ABO compatible & Yes & 60 & & 24 & & 0.32 \\
\cline { 2 - 7 } & No & 8 & & 8 & & \\
\hline
\end{tabular}

Graft dysfunction: The impact of infection on graft dysfunction was studied. Out of total of 68 patients of infection, 24 patients were excluded due to only rejection, while eight patients had concomitant rejection along with graft pyelonephritis and were included in the study. The mean Basal creatinine of the 22 patients who developed infection was $1.27+-0.24$, while the mean creatinine at the end of follow up was $1.51+-0.56$. On statistical analysis, a significant increase in creatinine at the end of one year was found in the infected group $(p=0.003)$.

Thirty-two patients had no episode of infection, out of which 10 developed rejection, and were excluded. We found that there was no significant increase in creatinine in the non infected group $(\mathrm{N}=22)$. However, on comparing the two groups [Table 3], there was no increase in creatinine at the end of the study period $(p>0.05)$.

Table 3: Comparison of Infected and Non-infected groups at one year

\begin{tabular}{|c|c|c|c|c|}
\hline Creatinine & $\mathrm{N}$ & Mean & S. D. & P value \\
\hline \multirow{2}{*}{ Basal } & 22 & 1.16 & 0.25 & 0.70 \\
\cline { 2 - 4 } & 44 & 1.20 & 0.26 & \\
\hline \multirow{2}{*}{ Final } & 22 & 1.27 & 0.24 & \multirow{2}{*}{0.12} \\
\cline { 2 - 4 } & 44 & 1.51 & 0.56 & \\
\hline \multirow{2}{*}{ Rise } & 22 & 0.11 & 0.20 & \multirow{2}{*}{0.14} \\
\cline { 2 - 4 } & 44 & 0.33 & 0.46 & \\
\hline
\end{tabular}

|www.worldwidejournals.com 


\section{DISCUSSION:}

Our study of major infections in the first year of transplant has reported an incidence of $68 \%$ which is similar to the reported data from the developing world[8,9].The majority of the infections was UTI(28\%); while out of the viral infections, it was Varicella zoster(8.7\%)[10,11].UTI is the commonest post transplant infection as reported by others with rates varying from $17 \%$ in USRDS data to $63 \%$ from the developing world. Our incidence is on the lower side inspite of universal DJ stent placement possibly due to early removal of urinary catheter[12]. The majority of the chicken pox infections took place in the two to six month period of transplantation, when the net state of immunosuppression is high. It was possibly due to one patient of chicken pox secondarily infecting others as the secondary attack rate of chicken pox is high (80\%).We found the incidence of CMV disease to be $4 \%$ which is lower as compared to previous studies possibly because of shorter follow up $[14,15]$.The reported prevalence of TB from developing countries $02-15 \%$ and we found an incidence of $7.1 \%$ which is similar to the reported literature. All patients were successfully treated with non Rifampicin based regimes[16].Hepatitis $\mathrm{C}$ infection has been reported to be a poor prognostic marker for graft survival, however we found no increase in rate of infection with Hepatitis $\mathbf{C}$ co-infection as we gave lesser immunosuppression to them[17].Tacrolimus and MMF based immunosuppression regimes have shown increased predisposition to infection. However, we found no difference between the two groups, possibly due to better pre-transplant screening and surveillance[16].We found $12 \%$ of patients with concomitant rejection/infection, leading to significant increase in serum creatinine. However on follow up at one year, it was statistically insignificant[13]. Our follow up has been only of one year and a longer follow up can give more definitive answers. We found an incidence of $4 \%$ death in our cohort of transplant patients, over a period of one year; which is possibly high as compared to the Western world, due to the socioeconomic and hygienic conditions prevalent in India[18].

\section{CONCLUSION}

Renal transplant programmes are on the rise in the developing world where the exposure to pathogen and socioeconomic conditions are likely to be different than the developed world. The etiological spectrum provides information to the treating clinician about the type of infections likely to be encountered.Infections cause transient graft dysfunction, however in the short term follow up, do not show an adverse outcome.

\section{REFERENCES:}

1. Chugh, K. (1994).Three decades of nephrology.J Postgrad Med,40(103).

2. Sia, I., \& Paya, C. (1998). Infectious complications following renal transplantation. Surg Clin North Am, 78(1), 95 - 112.

3. Alangaden, G., Thyagarajan, R., Gruber, S., Morawski, K., Garnick, J., \& ElAmm, J. et al. (2006). Infectious complications after kidney transplantation: current epidemiology and associated risk factors. Clinical Transplantation, 20(4), 401-409. doi: 10.1111/j.1399-0012.2006.00519.x

4. Maraha, B., Bonten, H., van Hooff, H., Fiolet, H., Buiting, A., \& Stobberingh, E. (2001). Infectious complications and antibiotic use in renal transplant recipients during a 1-year follow-up. Clinical Microbiology And Infection, 7(11),619-625. doi: 10.1046/j.1198-743x.2001.00329.x

5. John, G. (1999). Infections after renal transplantation in India. Transplantation Reviews, 13(4), 183-191.doi: 10.1016/s0955-470x(99)80082-2

6. Date, A., Vaska, K., Vaska, P., Pandey, A., Kirubakaran, M., \& Shastry, J. (1982). Terminal Infections in Renal Transplant Patients in a Tropical Environment. Nephron,32(3),253-257. doi: 10.1159/000182855

7. Briggs, J. (2001). Causes of death after renal transplantation. Nephrology Dialysis Transplantation, 16(8), 1545-1549. doi: 10.1093/ndt/16.8.1545

8. Gupta, R. (2007). Opportunistic Infections in Renal Allograft Recipients. Transplantation Proceedings, 39(3), 731-733. doi: 10. 1016/j. transproceed.2007.01.063

9. Rubin, R. (1993). Infectious disease complications of renal transplantation. Kidney International, 44(1),221-236. doi: 10.1038/ki.1993.234

10. Houry,J., \& Brennen, D. (2005). Infectious Complications in Kidney Transplant Recipients: A Review of Literature. Saudi J Kid Dis Transplant, 16(4), 453 - 497.

11. Morris, P., \& Knechtle, S. (2008). Kidney Transplantation: Principles and Practice (6th ed.). Saunders.

12. Dharnidharka, V., Agodoa, L., \& Abbott, K. (2007). Risk Factors for Hospitalization for Bacterial orViral Infection in Renal Transplant RecipientsAn Analysis of USRDS Data. American Journal Of Transplantation, 7(3), 653661.doi: $10.1111 /$ j.1600-6143.2006.01674.x
13. Chuang, P., Parikh, C., \& Langone, A. (2005). Urinary tract infections after renal transplantation: a retrospective review at two US transplant centers. Clinical Transplantation, 19(2),230-235. doi:10.1111/j.1399-0012.2005.00327.x

14. Gourishankar, S., McDermid, J., Jhangri, G., \& Preiksaitis, J. (2004). Herpes Zoster Infection Following Solid Organ Transplantation: Incidence, Risk Factors and Outcomes in the Current Immunosuppressive Era. American Journal Of Transplantation, 4(1), 108-115. doi: 10.1046/j.16006143.2003.00287.x

15. Sakhuja, V., Jha, V., Joshi, K., \& Nada, R. (2008). CMV disease among renal transplant recipients in India. Nephrology, 7(3), 125- 129.

16. Fietze, E., Prosch, S., Reinke, P., Stein, J., Docke, W., \& Staffa, G. et al. (1994). Cytomelagovirus Infection in Transplant Recipient The Role of Tumor Necrosis Factor. Transplantation, 58(6), 675-680. doi: 10.1097/00007890199409000-00007

17. Sakhuja,V., Jha, V.,Varma, P., Joshi, K., \& Chugh, K. (1996).The High Incidence of Tuberculosis Among Renal Transplant Recipients in India. Transplantation, 61(2),211-215. doi: 10.1097/00007890-199601270-00008

18. Ingsathit, A., Thakkinstian, A., Kantachuvesiri, S., \& Sumethkul, V. (2007) Different Impacts of Hepatitis B Virus and Hepatitis CVirus on the Outcome of Kidney Transplantation. Transplantation Proceedings, 39(5), 1424-1428. doi: 10.1016/j.transproceed.2007.02.068 